Ecología

\title{
Composición y estructura del ensamble de tenebriónidos epigeos (Coleoptera: Tenebrionidae) de ecosistemas continentales e insulares del desierto costero transicional de Chile
}

\section{Composition and structure of the epigean tenebrionid assemblage (Coleoptera: Tenebrionidae) of continental and insular ecosystems of the transitional coastal desert of Chile}

\author{
Fermín M. Alfaro $^{\mathrm{a}, \mathrm{b}}$, Jaime Pizarro-Araya ${ }^{\mathrm{a}, \mathrm{b}, *}$ y Gustavo E. Flores ${ }^{\mathrm{c}}$ \\ ${ }^{a}$ Laboratorio de Entomología Ecológica, Departamento de Biología, Universidad de La Serena, Casilla 554, La Serena, Chile \\ ${ }^{\mathrm{b}}$ Instituto de Investigación Multidisciplinar en Ciencia y Tecnología, Universidad de La Serena, Casilla 554, La Serena, Chile \\ ${ }^{\mathrm{c}}$ Laboratorio de Entomología, Instituto Argentino de Investigaciones de las Zonas Áridas (CCT CONICET, Mendoza), Casilla de correo 507, 5500 Mendoza, \\ Argentina
}

Recibido el 4 de marzo de 2016; aceptado el 30 de mayo de 2016

Disponible en Internet el 31 de octubre de 2016

\begin{abstract}
Resumen
Se estudió la composición taxonómica, riqueza, abundancia y estructura espacio-temporal del ensamble de tenebriónidos epigeos en diferentes hábitats continentales e insulares del desierto costero transicional de Chile. Los muestreos se realizaron en el borde continental de Punta de Choros y en el archipiélago de Los Choros durante los años 2005 y 2006 mediante el uso de trampas de intercepción. Se capturó un total de 982 individuos pertenecientes a 7 tribus, 9 géneros y 17 especies, siendo Praocis Eschscholtz y Gyriosomus Guérin-Méneville los géneros más diversos. Se realizó el análisis Anosim que encontró diferencias significativas entre estaciones $(\mathrm{R}=0.31 ; p=0.01)$ pero no entre hábitats $(\mathrm{R}=0.1872 ; p=0.12)$, aunque con un bajo efecto de estos factores. La mayor riqueza y diversidad se observó en los hábitats estepa costera interior y estepa costera Choros, mientras que la mayor abundancia se registró en estepa costera interior (26.5\%). No se observó una estructura estacional, pero los datos mostraron una mayor abundancia en primavera tanto en el continente como en las islas, a excepción de la isla Choros, donde la mayor abundancia se registró en invierno. Se discuten estas diferencias en relación con las características pedológicas y vegetacionales de los ambientes estudiados. (C) 2016 Universidad Nacional Autónoma de México, Instituto de Biología. Este es un artículo Open Access bajo la licencia CC BY-NC-ND (http://creativecommons.org/licenses/by-nc-nd/4.0/).
\end{abstract}

Palabras clave: Diversidad; Desierto costero; Islas; Tenebrionidae; Reserva Nacional Pingüino de Humboldt

\section{Abstract}

Pitfall traps were used to study the taxonomic composition, richness, abundance, and spatio-temporal structure of the assemblage of epigean tenebrionids in continental and insular habitats of the transitional coastal desert of Chile. Samplings were conducted on the coastal area of Punta de Choros and in the Archipelago Los Choros in 2005 and 2006. A total of 982 specimens were captured, belonging to 7 tribes, 9 genera, and 17 species. The most diverse genera were Praocis Eschscholtz and Gyriosomus Guérin-Méneville. The Anosim analysis showed statistically-significant differences between seasons $(\mathrm{R}=0.31 ; p=.01)$ but not among habitats $(\mathrm{R}=0.1872 ; p=.12)$, although the effect of these factors was low. The highest richness and diversity was observed in the interior coastal steppe and the coastal steppe of Los Choros, whereas the highest abundance occurred in the interior coastal steppe (26.5\%). Seasonal structure was not observed; however, the data showed

\footnotetext{
* Autor para correspondencia.

Correo electrónico: japizarro@userena.cl (J. Pizarro-Araya).

La revisión por pares es responsabilidad de la Universidad Nacional Autónoma de México.
} 
increased abundance in spring both in the continent and on the islands, with the exception of Choros island, where the highest abundance occurred in winter. This paper discusses the differences in the soil and plant characteristics of the ecosystems under study.

(C) 2016 Universidad Nacional Autónoma de México, Instituto de Biología. This is an open access article under the CC BY-NC-ND license (http://creativecommons.org/licenses/by-nc-nd/4.0/).

Keywords: Diversity; Coastal desert; Islands; Tenebrionidae; Pingüino de Humboldt National Reserve

\section{Introducción}

El desierto costero transicional de Chile $\left(25^{\circ}-32^{\circ} \mathrm{S}\right)$ comprende parte de la provincia del desierto costero peruano (Morrone, 2015) y se caracteriza por representar el límite septentrional del hotspot Chileno (Gaston, 2000) y por la presencia de una biota particular en términos de diversidad y endemismo vegetacional (Armesto, Vidiella y Gutiérrez,1993; Cavieres et al., 2001; Cavieres et al., 2002). El conocimiento de coleópteros del desierto costero transicional de Chile se ha centrado principalmente en aspectos taxonómicos y sistemáticos (Elgueta, 2013; Flores y Pizarro-Araya, 2012, 2014; Jerez, 2000; Pizarro-Araya, Cepeda-Pizarro y Flores, 2008). Los estudios ecológicos se han centrado en la dinámica poblacional de artrópodos epigeos (Cepeda-Pizarro, Pizarro-Araya y Vásquez, 2005a, b; Cortés-Contreras, Pizarro-Araya, Alfaro y Cepeda-Pizarro, 2013) y otros trabajos se han enfocado en áreas continentales e insulares del desierto costero del Huasco, franja del desierto costero transicional de Chile característica en términos vegetacionales (Gajardo, 1993). Particularmente para las áreas insulares se han descrito especies endémicas (Grismado y Pizarro-Araya, 2016; Pizarro-Araya y Flores, 2004), se ha documentado la diversidad de insectos (Alfaro, Pizarro-Araya y Flores, 2009; Pizarro-Araya, Alfaro et al., 2014) y se han estudiado las variaciones morfológicas de coleópteros tenebriónidos (Benítez, Pizarro-Araya, Bravi, Sanzana y Alfaro, 2014).

Uno de los grupos de artrópodos característico de los ecosistemas áridos y semiáridos lo representa la familia Tenebrionidae (Matthews, Lawrence, Bouchard, Steiner y Ślipiński, 2010). Estos insectos poseen adaptaciones morfológicas, fisiológicas y etológicas para la vida en estos ambientes (CloudsleyThompson, 2001; Duncan, Dickman y Christopher, 2009) y cumplen un papel clave en los procesos de fragmentación del recurso vegetal, en los ciclos de los nutrientes y en las tramas tróficas, principalmente como componentes de la dieta de otros organismos consumidores (Vidal, Pizarro-Araya, Jerez y Ortiz, 2011). Al presente no se ha evaluado si los hábitats costeros de la Región de Coquimbo (Chile) presentan diferencias sobre los patrones de diversidad y abundancia de tenebriónidos epigeos y no se conoce el grado de estructuración de las comunidades de tenebriónidos epigeos continentales e insulares del borde costero de la región. Considerando estos antecedentes, los objetivos de este trabajo son: 1) describir la composición taxonómica, riqueza y abundancia de tenebriónidos epigeos en hábitats continentales e insulares y 2) comparar la estructura del ensamble de tenebriónidos epigeos entre estaciones y hábitats continentales e insulares.

\section{Materiales y métodos}

Los muestreos se realizaron en diferentes hábitats ubicados tanto en la franja costera continental de Punta de Choros como en el archipiélago de Los Choros (Región de Coquimbo). Para el continente se seleccionaron 4 sitios de muestreo, que corresponden a distintos tipos de hábitats: estepa costera (EC; $29^{\circ} 15^{\prime} 12^{\prime \prime} \mathrm{S}, 71^{\circ} 26^{\prime} 59^{\prime \prime} \mathrm{O}, 17 \mathrm{~m} \mathrm{snm}$ ), duna costera (DC; $29^{\circ} 16^{\prime} 31^{\prime \prime} \mathrm{S}, 7^{\circ} 23^{\prime} 51^{\prime \prime} \mathrm{O}, 18 \mathrm{~m} \mathrm{snm}$ ), humedal costero (HC; $29^{\circ} 18^{\prime} 18^{\prime \prime} \mathrm{S}, 71^{\circ} 21^{\prime} 50^{\prime \prime} \mathrm{O}, 2 \mathrm{~m}$ snm) y estepa costera interior (ECI; $29^{\circ} 19^{\prime} 48^{\prime \prime} \mathrm{S}, 71^{\circ} 19^{\prime} 46^{\prime \prime} \mathrm{O}, 23 \mathrm{~m}$ snm). Para cada isla se seleccionaron 3 sitios de muestreo que corresponden a 3 tipos de hábitats: isla Choros: estepa costera Choros (ECCh; $29^{\circ} 32^{\prime} \mathrm{S}, 67^{\circ} 61^{\prime} \mathrm{O}$ ); pedregoso interior Choros (PICh; 292 $8^{\prime} \mathrm{S}, 67^{\circ} 59^{\prime} \mathrm{O}$ ) y pedregoso costero Choros (PCCh; $29^{\circ} 29^{\prime} \mathrm{S}, 67^{\circ} 58^{\prime}$ O). Isla Damas: estepa costera norte Damas (ECND; $29^{\circ} 13^{\prime} \mathrm{S}, 71^{\circ} 31^{\prime} \mathrm{O}$ ); estepa costera interior Damas (EID; $29^{\circ} 14^{\prime} \mathrm{S}, 71^{\circ} 31^{\prime} \mathrm{O}$ ) y estepa costera sur Damas (ECSD; $29^{\circ} 14^{\prime} \mathrm{S}, 71^{\circ} 31^{\prime} \mathrm{O}$ ). Isla Gaviota: duna costera Gaviota (DCG; $29^{\circ} 15^{\prime} \mathrm{S}, 71^{\circ} 28^{\prime} \mathrm{O}$ ); duna interior Gaviota (DIG; $29^{\circ} 15^{\prime} \mathrm{S}$, $\left.71^{\circ} 28^{\prime} \mathrm{O}\right)$ y estepa costera Gaviota (ECG; $29^{\circ} 15^{\prime} \mathrm{S}, 71^{\circ} 26^{\prime} \mathrm{O}$ ) (fig. 1).

El clima del área de estudio presenta tendencia mediterránea (Di Castri y Hajek, 1976). Las temperaturas son bajas, al igual que la amplitud térmica diaria y anual producto de la influencia marina (Armesto et al., 1993). La precipitación anual promedio del área es $\sim 90 \mathrm{~mm}$; con años secos y lluviosos que ocurren en ciclos irregulares relacionados con el evento El Niño Oscilación del Sur (Novoa y Villaseca, 1989). Los antecedentes geomorfológicos y biofísicos del área de estudio se describen en Aguirre (1967) y Castro y Brignardello (2005). La diversidad florística del archipiélago se detalla en Arancio y Jara (2007).

En cada sitio de muestreo (continental e insular) se instalaron 20 trampas de intercepción dispuestas en parcelas de $4 \times 5 \mathrm{~m}$ ubicando 2 parcelas (réplicas) por cada hábitat de acuerdo con Cepeda-Pizarro et al. (2005a, b). Las trampas operaron 3 días durante cada uno de los 3 meses (agosto, octubre, diciembre) de 2005 y 2006. El material capturado fue retirado, limpiado y conservado en alcohol (70\%) hasta el momento de su procesamiento y montaje. Para la identificación taxonómica a nivel genérico y específico se utilizó literatura especializada. El material colectado está depositado en la colecciones del Laboratorio de Entomología Ecológica, Universidad de La Serena, Chile (LEULS) y del Laboratorio de Entomología, Instituto Argentino de Investigaciones de Zonas Áridas, Mendoza, Argentina (IADIZA). 

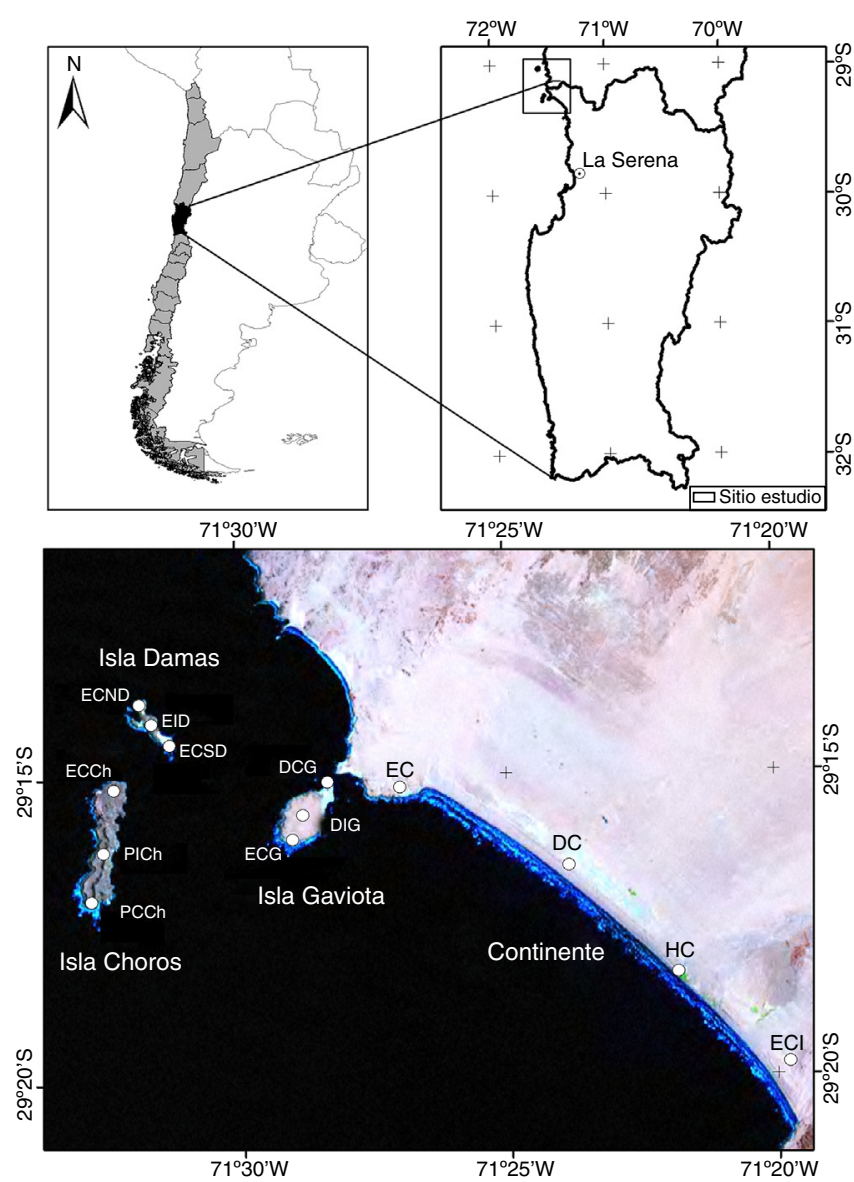

Figura 1. Localización geográfica del área de estudio. Continente: EC (estepa costera), DC (duna costera), $\mathrm{HC}$ (humedal costero), ECI (estepa costera interior); isla Choros: ECCh (estepa costera Choros), PICh (pedregoso interior Choros), PCCh (pedregoso costero Choros); isla Damas: ECND (estepa costera norte Damas), EID (estepa costera interior Damas), ECSD (estepa costera sur Damas); isla Gaviota: DCG (duna costera Gaviota), DIG (duna interior Gaviota), ECG (estepa costera Gaviota).

Para cada uno de los hábitats estudiados se estimó la riqueza de especies, abundancia y diversidad según el índice de Shannon-Weaver (H') (Shannon y Weaver, 1949). Para evaluar la riqueza taxonómica del ensamble de tenebriónidos epigeos y el esfuerzo de muestreo se construyeron curvas de rarefacción de especies (Gotelli y Colwell, 2010).

Para analizar el nivel de estructura del ensamble de tenebriónidos epigeos se realizó un análisis de escalamiento multidimensional no métrico (nMDS) (Clarke, 1993; Field, Clarke y Warwick, 1982) utilizando las matrices de similitud de Bray-Curtis y Jaccard. Los criterios de agrupación se estimaron de acuerdo con la gráfica de la envolvente convexa y el índice de stress de Kruskal (Johnson y Wichern, 1992; Kruskal, 1964).

Para analizar el grado de estructuración estacional y por hábitat se realizó un análisis de similitud por agrupamientos (cluster) sobre las matrices de similitud de Bray-Curtis y Jaccard (Faith, Minchin y Belbin, 1987). Para evaluar la significación estadística de las diferencias entre las estaciones y hábitats se realizó un análisis de similitudes (Anosim) de una vía basado en la distancia de los índices de Bray-Curtis y de Jaccard. El nivel de significación se evaluó mediante 50,000 permutaciones. Posteriormente

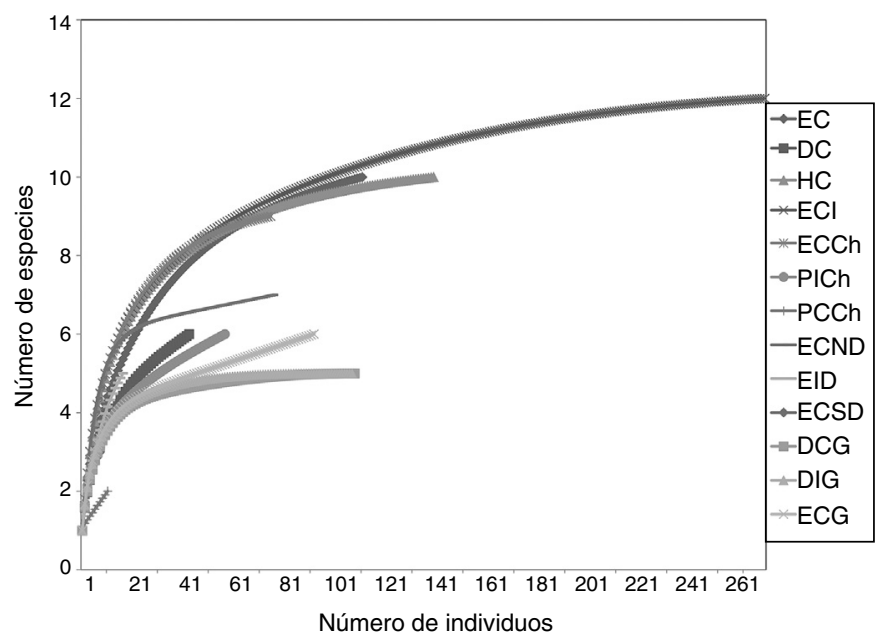

Figura 2. Curva de acumulación de especies de tenebriónidos epigeos en diferentes hábitats continentales e insulares del desierto costero transicional de Chile. DC: duna costera; DCG: duna costera Gaviota; DIG: duna interior Gaviota; EC: estepa costera; ECCh: estepa costera Choros; ECG: estepa costera Gaviota; ECI: estepa costera interior; ECND: estepa costera norte Damas; ECSD: estepa costera sur Damas; EID: estepa costera interior Damas; HC: humedal costero; PCCh: pedregoso costero Choros; PICh: pedregoso interior Choros.

se realizaron pruebas pareadas con Anosim utilizando valores de probabilidad $(p)$ corregidos por el método de Bonferroni. Todos los análisis fueron realizados mediante el programa PAST 2.16 (Hammer, Harper y Ryan, 2001).

\section{Resultados}

\section{Composición del ensamble de tenebriónidos epigeos}

Se capturó un total de 982 individuos agrupados en 7 tribus, 9 géneros y 17 especies para toda el área de estudio (tabla 1). Los géneros más diversos fueron Praocis Eschscholtz (4 especies) y Gyriosomus Guérin-Méneville (6 especies). La mayor riqueza de especies se observó en ECI (con 11 especies) y ECCh (con 9 especies). Asimismo, la diversidad de Shannon-Weaver fue mayor tanto en ECI $\left(\mathrm{H}^{\prime}=1.86\right)$ como en ECCh $\left(\mathrm{H}^{\prime}=1.80\right)$ (tabla 1). Del total de 17 especies registradas solo 6 fueron compartidas entre el continente y las islas; estas fueron: Praocis (P.) spinolai Gay y Solier, P. (Mesopraocis) pilula Laporte, $P$. (Postpraocis) curtisi Solier, Diastoleus girardi Peña, Scotobius bullatus Curtis y Nycterinus (Paranycterinus) rugiceps Curtis. Las curvas de rarefacción independientes para los 13 hábitats estudiados mostraron diferentes niveles de acumulación de especies, siendo ECI el que presentó la curva con mayor número de especies sin llegar a estabilizarse. Solo la curva de acumulación de DIG se estabilizó. Las curvas de rarefacción de ECCh, ECND, HC, EID, EC, PICh, ECG, DCG, DC y PCCh no llegaron a estabilizarse (fig. 2).

Las especies registradas solo en el continente fueron Gyriosomus whitei Waterhouse, Entomochilus freudei Peña, Praocis (Orthogonoderes) chilensis (Gray) y Psammetichus pilipes Guérin-Méneville, mientras que las especies observadas solo en islas fueron Entomochilus pilosus (Solier), Entomochilus sp., Thinobatis melcheri Freude, Thinobatis simplex Peña y 
Tabla 1

Diversidad del ensamble de tenebriónidos epigeos en hábitats pedológicos continentales e insulares del desierto costero transicional de Chile.

\begin{tabular}{|c|c|c|c|c|c|c|c|c|c|c|c|c|c|c|c|c|}
\hline \multirow[b]{3}{*}{ Tribu } & \multirow[b]{3}{*}{ Especies } & \multicolumn{4}{|c|}{ Continente } & \multicolumn{9}{|c|}{ Archipiélago } & \multicolumn{2}{|c|}{ Total } \\
\hline & & \multirow[b]{2}{*}{$\mathrm{EC}$} & \multirow[b]{2}{*}{ DC } & \multirow[b]{2}{*}{$\mathrm{HC}$} & \multirow[b]{2}{*}{ ECI } & \multicolumn{3}{|c|}{ Isla Choros } & \multicolumn{3}{|c|}{ Isla Damas } & \multicolumn{3}{|c|}{ Isla Gaviota } & \multirow[b]{2}{*}{$\mathrm{n}$} & \multirow[b]{2}{*}{$\%$} \\
\hline & & & & & & $\mathrm{ECCh}$ & $\mathrm{PICh}$ & PCCh & ECND & EID & ECSD & DCG & DIG & ECG & & \\
\hline \multirow[t]{4}{*}{ Nycteliini } & Gyriosomus elongatus Waterhouse, 1843 & 0 & 0 & 10 & 83 & 0 & 0 & 0 & 0 & 0 & 2 & 0 & 0 & 0 & 95 & 9.7 \\
\hline & G. granulipennis Pizarro-Araya y Flores, 2004 & 0 & 0 & 0 & 0 & 14 & 1 & 0 & 0 & 0 & 0 & 0 & 0 & 0 & 15 & 1.5 \\
\hline & G. whitei Waterhouse, 1844 & 3 & 0 & 26 & 1 & 0 & 0 & 0 & 0 & 0 & 0 & 0 & 0 & 0 & 30 & 3.1 \\
\hline & Psectrascelis elongata Solier, 1851 & 1 & 0 & 0 & 0 & 0 & 0 & 0 & 0 & 0 & 0 & 2 & 0 & 0 & 3 & 0.3 \\
\hline \multirow[t]{3}{*}{ Physogasterini } & Entomochilus freudei Peña, 1980 & 0 & 0 & 0 & 3 & 0 & 0 & 0 & 0 & 0 & 0 & 0 & 0 & 0 & 3 & 0.3 \\
\hline & E. pilosus (Solier, 1844) & 0 & 0 & 0 & 0 & 0 & 0 & 0 & 0 & 0 & 0 & 0 & 0 & 1 & 1 & 0.1 \\
\hline & Entomochilus sp. & 0 & 0 & 0 & 0 & 4 & 0 & 0 & 0 & 0 & 0 & 0 & 0 & 0 & 4 & 0.4 \\
\hline \multirow[t]{4}{*}{ Praociini } & Praocis (Praocis) spinolai Gay y Solier, 1840 & 0 & 0 & 0 & 50 & 25 & 10 & 10 & 7 & 0 & 0 & 17 & 14 & 12 & 145 & 14.8 \\
\hline & P. (Mesopraocis) pilula Laporte, 1840 & 36 & 3 & 20 & 9 & 1 & 0 & 0 & 14 & 2 & 1 & 13 & 3 & 0 & 102 & 10.4 \\
\hline & P. (Postpraocis) curtisi Solier, 1851 & 4 & 14 & 0 & 21 & 3 & 0 & 0 & 10 & 1 & 0 & 11 & 8 & 0 & 72 & 7.3 \\
\hline & P. (Orthogonoderes) chilensis (Gray, 1832) & 2 & 2 & 1 & 58 & 0 & 0 & 0 & 0 & 0 & 0 & 0 & 0 & 0 & 63 & 6.4 \\
\hline Elenophorini & Psammetichus pilipes Guérin-Méneville, 1834 & 0 & 0 & 0 & 3 & 0 & 0 & 0 & 0 & 0 & 0 & 0 & 0 & 0 & 3 & 0.3 \\
\hline \multirow[t]{2}{*}{ Thinobatini } & Thinobatis melcheri Freude, 1960 & 0 & 0 & 0 & 0 & 0 & 0 & 0 & 0 & 3 & 0 & 0 & 0 & 0 & 3 & 0.3 \\
\hline & T. simplex Peña, 1974 & 0 & 0 & 0 & 0 & 3 & 29 & 0 & 1 & 0 & 0 & 0 & 0 & 24 & 57 & 5.8 \\
\hline \multirow[t]{2}{*}{ Scotobiini } & Diastoleus girardi Peña, 1980 & 0 & 0 & 3 & 2 & 16 & 1 & 0 & 11 & 0 & 0 & 0 & 0 & 1 & 34 & 3.5 \\
\hline & Scotobius bullatus Curtis, 1845 & 4 & 1 & 7 & 13 & 2 & 13 & 0 & 16 & 1 & 5 & 0 & 25 & 9 & 96 & 9.8 \\
\hline \multirow[t]{4}{*}{ Eleodini } & Nycterinus (Paranycterinus) rugiceps Curtis, 1845 & 25 & 0 & 9 & 17 & 7 & 3 & 1 & 17 & 10 & 0 & 65 & 57 & 45 & 256 & 26.1 \\
\hline & Abundancia relativa $(\mathrm{N})$ & 75 & 20 & 76 & 260 & 75 & 57 & 11 & 76 & 17 & 8 & 108 & 107 & 92 & 982 & 100 \\
\hline & Número de especies (S) & 7 & 4 & 7 & 11 & 9 & 6 & 2 & 7 & 5 & 3 & 5 & 5 & 6 & 17 & \\
\hline & Diversidad de Shannon-Weaver $\left(H^{\prime}\right)$ & 1.58 & 1.17 & 1.79 & 1.86 & 1.80 & 1.28 & 0.30 & 1.80 & 1.20 & 0.90 & 1.16 & 1.24 & 1.29 & & \\
\hline
\end{tabular}

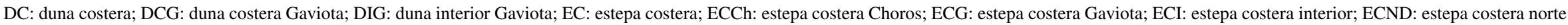

Damas; ECSD: estepa costera sur Damas; EID: estepa costera interior Damas; HC: humedal costero; PCCh: pedregoso costero Choros; PICh: pedregoso interior Choros. 

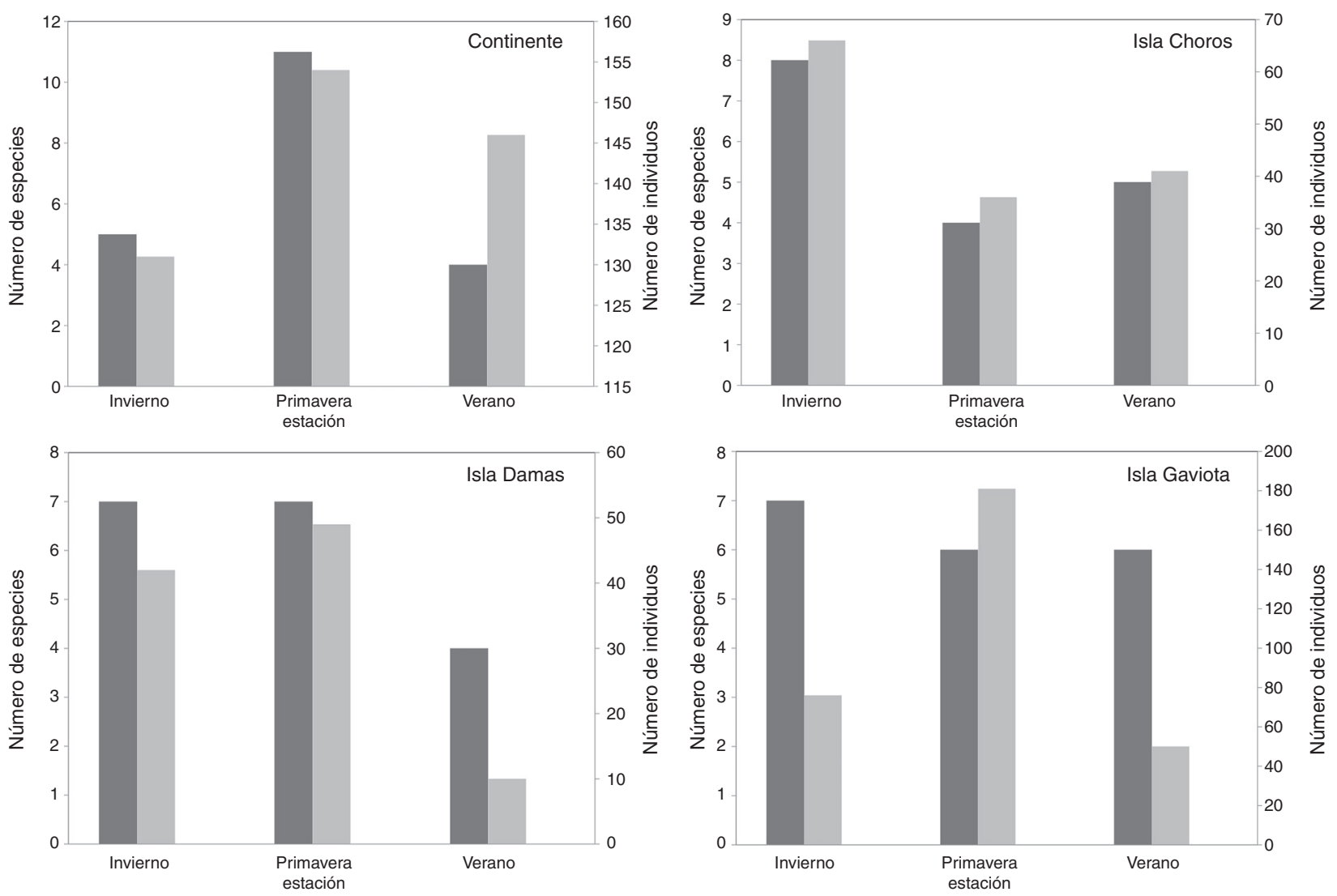

Figura 3. Riqueza (número de especies) y abundancia relativa (número de individuos) del ensamble de tenebriónidos epigeos durante 3 estaciones de muestreo en hábitats continentales e insulares del desierto costero transicional de Chile.

Gyriosomus granulipennis Pizarro-Araya y Flores, esta última endémica de la isla Choros (tabla 1). De acuerdo con las estaciones climáticas, la mayor riqueza en el continente se observó en primavera con 11 especies; en las islas Choros y Gaviota se registró en invierno con 8 y 7 especies, respectivamente, mientras que en la isla Damas se observó tanto en invierno como en primavera con 7 especies. La menor riqueza se registró en verano tanto en el continente (4 especies) como en las islas Damas (4 especies) y Gaviota (6 especies; fig. 3).

Abundancia relativa del ensamble de tenebriónidos epigeos. La mayor abundancia se observó en las islas con el 56.1\% del total capturado, mientras que el continente representó el $43.9 \%$. Dentro del archipiélago, el aporte al total capturado estuvo representado por isla Gaviota (31.3\%), isla Choros (14.6\%) e isla Damas (10.3\%). Los hábitats con mayor aporte al total capturado en ambientes de continente e islas fueron ECI (26.5\%), DCG (11.0\%), DIG (10.9\%) (fig. 4). Entre los hábitats insulares estudiados, la mayor abundancia se observó en DCG (19.6\% del total capturado en islas) y DIG (19.4\%), ambos ambientes dunarios de la isla Gaviota, mientras que en el continente se registró en ECI (60.3\% del total capturado en el continente). Los hábitats con menor aporte al total capturado en islas fueron ECSD (1.5\%) y PCCh (2.0\%), mientras que en el continente lo fue DC (4.6\%). Las especies más abundantes del ensamble fueron Nycterinus (Paranycterinus) rugiceps Curtis (26.1\% del total capturado) y
Praocis (P.) spinolai Gay y Solier (14.8\%), la primera presente en todos los hábitats continentales e insulares (tabla 1).

Considerando las estaciones climáticas muestreadas, la mayor abundancia se observó en primavera tanto en el continente como en islas, a excepción de la isla Choros, donde el mayor aporte se registró en invierno (fig. 3). La estación con el menor aporte al total capturado fue invierno en el continente, primavera en isla Choros y verano en las islas Damas y Gaviota (fig. 3).

Estructura del ensamble de tenebriónidos epigeos. El Anosim mediante la matriz de Bray-Curtis y la matriz de Jaccard encontró diferencias significativas entre estaciones $(\mathrm{R}=0.31$; $p=0.01)(\mathrm{R}=0.2568 ; p=0.01)$, pero no entre hábitats muestrea$\operatorname{dos}(\mathrm{R}=0.1872 ; p=0.12)(\mathrm{R}=0.2705 ; p=0.05)$. Sin embargo, estas diferencias no dan cuenta de una clara estructura debido a valores de $\mathrm{R}$ bajos.

El análisis de cluster mediante la matriz de Bray-Curtis no mostró agrupaciones significativas entre los hábitats estudiados. Solo se observó un grupo (sobre un $70 \%$ de similitud) de hábitats de dunas costeras pertenecientes a la isla Gaviota (fig. 5).

El análisis de escalamiento multidimensional no-métrico sugiere que el ensamble de tenebriónidos epigeos presentaría estructura principalmente en hábitats de estepa, con una alta superposición de comunidades entre diferentes sitios mayoritariamente costeros (fig. 6). 


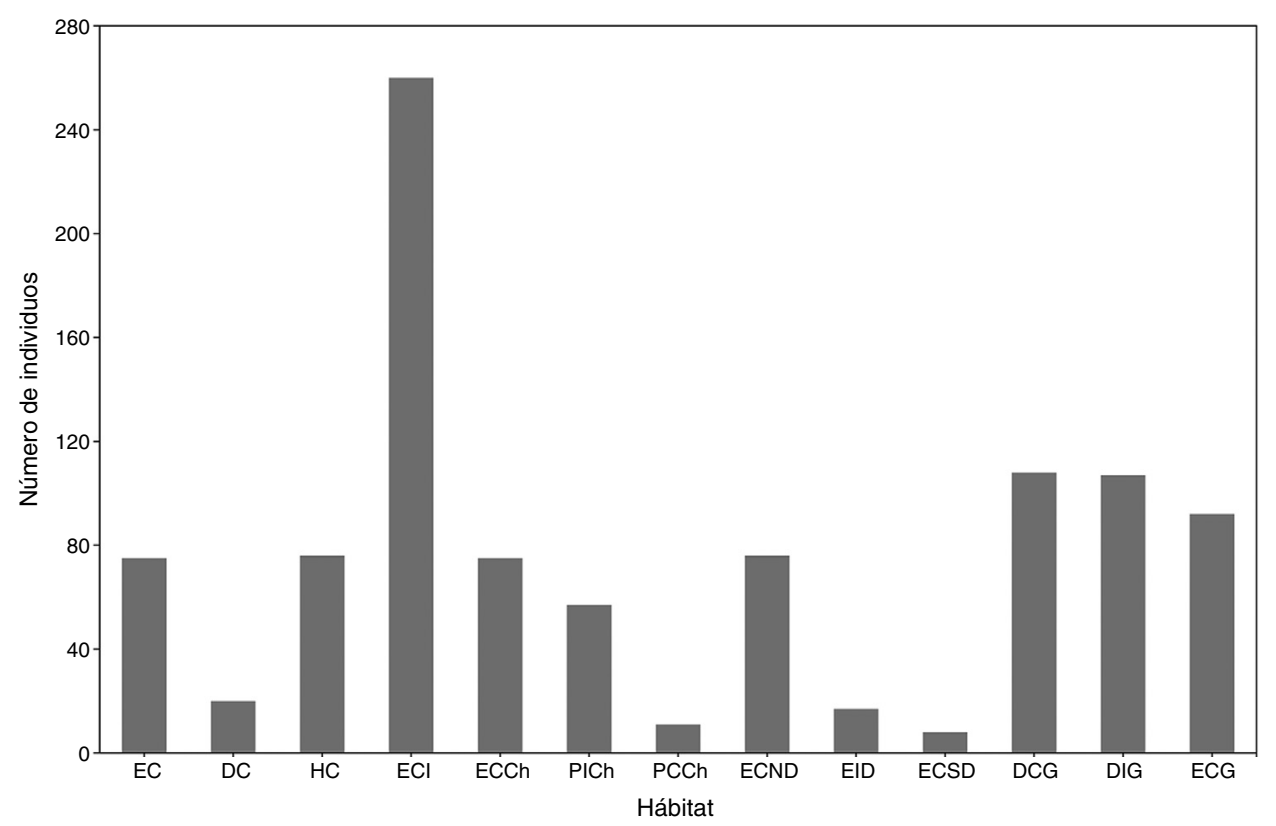

Figura 4. Abundancia relativa (número de individuos) del ensamble de tenebriónidos epigeos presentes en hábitats continentales e insulares del desierto costero transicional de Chile. DC: duna costera; DCG: duna costera Gaviota; DIG: duna interior Gaviota; EC: estepa costera; ECCh: estepa costera Choros; ECG: estepa costera Gaviota; ECI: estepa costera interior; ECND: estepa costera norte Damas; ECSD: estepa costera sur Damas; EID: estepa costera interior Damas; HC: humedal costero; PCCh: pedregoso costero Choros; PICh: pedregoso interior Choros.

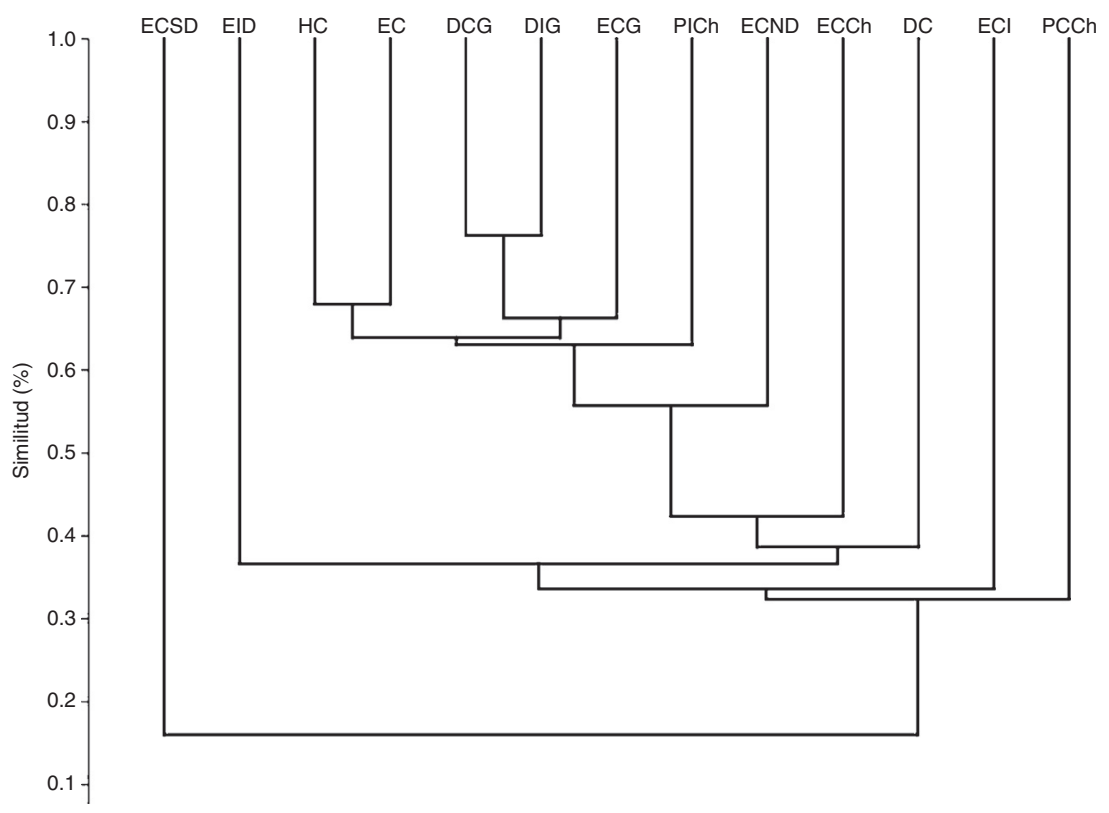

Figura 5. Cluster de similitud basado en el índice de Bray-Curtis para los hábitats estudiados. DC: duna costera; DCG: duna costera Gaviota; DIG: duna interior Gaviota; EC: estepa costera; ECCh: estepa costera Choros; ECG: estepa costera Gaviota; ECI: estepa costera interior; ECND: estepa costera norte Damas; ECSD: estepa costera sur Damas; EID: estepa costera interior Damas; HC: humedal costero; PCCh: pedregoso costero Choros; PICh: pedregoso interior Choros.

\section{Discusión}

El ensamble de tenebriónidos epigeos estuvo representado por 17 especies, que en su mayoría presentan distribución amplia en la cordillera de la costa de la Región de Coquimbo (Cortés-Contreras et al., 2013), con excepción de Gyriosomus granulipennis, que es endémica de la isla Choros (Alfaro et al., 2009; Pizarro-Araya, Vergara y Flores, 2012) y recientemente ha sido categorizada como «vulnerable» por el Ministerio de
Medio Ambiente (2011), e incorporada en la Ley de caza y su reglamento (SAG, 2015).

Las curvas de rarefacción para los distintos hábitats estudiados presentaron en su mayoría baja tendencia a la asíntota, lo que sugiere que el esfuerzo de muestreo no sería suficiente para describir el número de especies del ensamble de tenebriónidos epigeos. Sin embargo, los datos muestran que la mayor riqueza de especies y los mayores valores de Shannon-Weaver se registraron en ECI, sitio correspondiente a ambientes de EC 

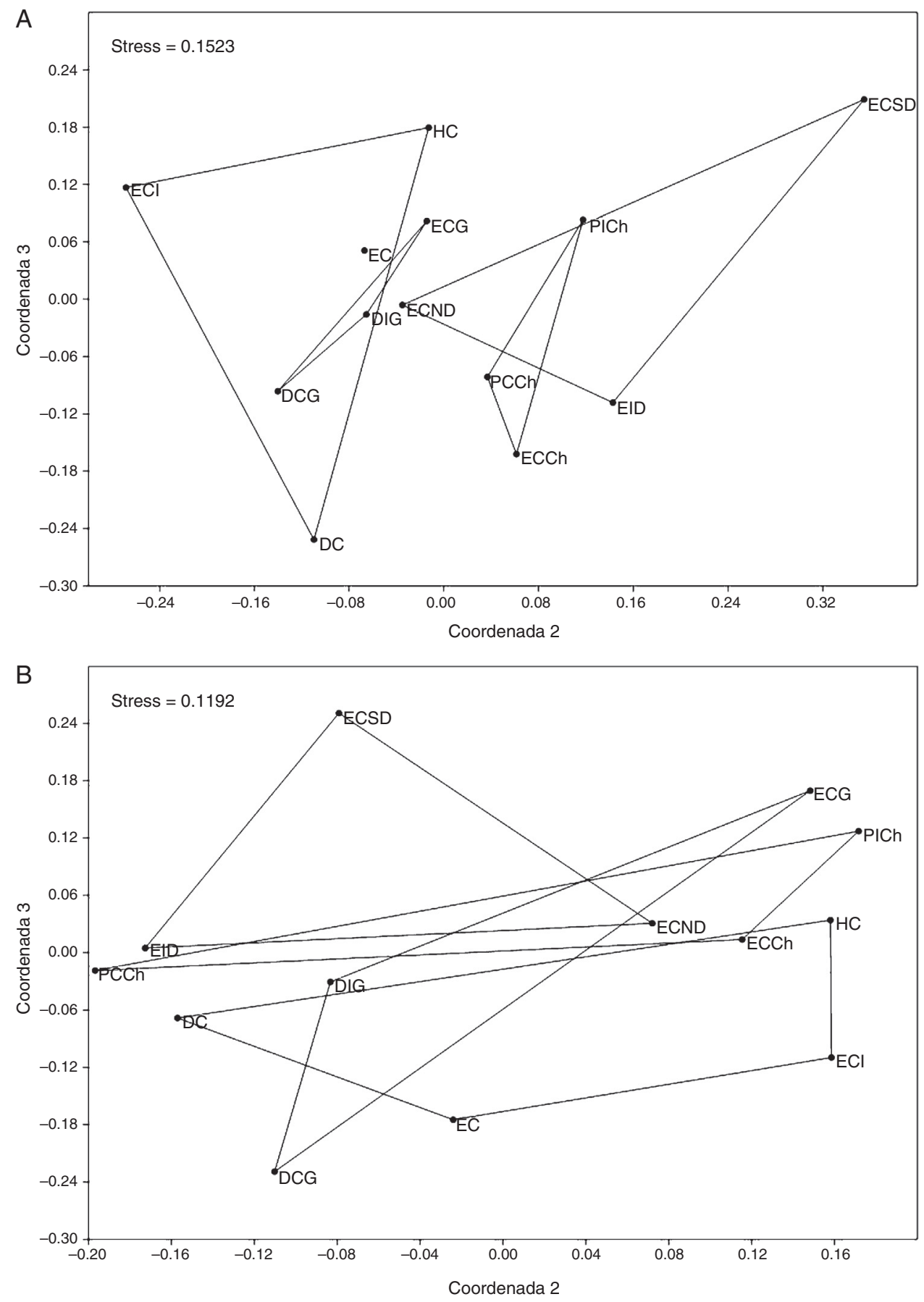

Figura 6. Análisis de escalamiento multidimensional no-métrico (nMDS) y valor de stress de Kruskal: A, basado sobre el índice de similitud de Bray-Curtis; B, basado sobre el índice de similitud de Jaccard. DC: duna costera; DCG: duna costera Gaviota; DIG: duna interior Gaviota; EC: estepa costera; ECCh: estepa costera Choros; ECG: estepa costera Gaviota; ECI: estepa costera interior; ECND: estepa costera norte Damas; ECSD: estepa costera sur Damas; EID: estepa costera interior Damas; HC: humedal costero; PCCh: pedregoso costero Choros; PICh: pedregoso interior Choros.

que sostienen una importante riqueza de artrópodos epigeos (Pizarro-Araya, Agusto et al., 2014; Valdivia, Pizarro-Araya, Briones, Ojanguren-Affilastro y Cepeda-Pizarro, 2011).

El análisis de ordenamiento mostró una tendencia a agrupar hábitats mayoritariamente del tipo EC de isla Damas, los cuales se separaron de hábitats pertenecientes al continente. Asimismo, ambientes pertenecientes a la isla Choros se diferenciaron de aquellos ambientes de la costa continental. $\mathrm{Al}$ respecto en isla
Choros predominan los ambientes de tipo pedregoso, a diferencia de aquellos presentes en el continente que poseen suelos arenosos (Aguirre, 1967).

Los análisis de similitud mostraron que la abundancia del ensamble de tenebriónidos no presenta claras diferencias entre las estaciones muestreadas. Nuestros datos muestran fluctuaciones en la abundancia total del ensamble de tenebriónidos epigeos, lo que sugiere que la mayoría de estas especies poseen 
una denso-actividad condicionada por la disponibilidad de vegetación y humedad del suelo. Tanto la abundancia como la distribución espacial de tenebriónidos de sistemas áridos y semiáridos pueden estar limitadas e influenciadas por el tipo de suelo y vegetación (Santos, Gómez-González, Alonso, Arbelo y de Nicolás, 2000; Pan et al., 2015). La preferencia de hábitats de estos insectos se relaciona con las características del sustrato, por lo que suelos arenosos representan condiciones ideales para la ovipostura, desarrollo hipogeo de los estadios larvarios, obtención de alimentos (e.g., raíces y bulbos de plantas anuales y geófitas), excavación y conductas evasivas a altas temperaturas (Pizarro-Araya, 2010), en comparación con suelos de textura compacta y pedregosa (Parmenter y MacMahon, 1984). Factores como las precipitaciones y temperaturas pueden condicionar la disponibilidad de humedad del suelo y por tanto ser factores limitantes en esos hábitats costeros (Fattorini, 2009; Parmenter, Parmenter y Cheney, 1989).

Para otras latitudes se ha evaluado la importancia de las variables ecogeográficas (i.e., área, latitud, longitud, cercanía entre islas, distancia desde el continente) sobre la riqueza específica y los niveles de endemismo en los ensambles de tenebriónidos (Fattorini, 2002), así como también la importancia de la paleogeografía y el clima en la composición de estos ensambles (Santos, de Nicolás y Ferrer, 2002; Fattorini, 2006; Hausdorf y Henning, 2005). Nuestros datos sugieren que tanto los ambientes continentales como los insulares presentan pequeñas diferencias en la composición y abundancia del ensamble de tenebriónidos epigeos, lo que estaría dando cuenta de un área con características pedológicas, vegetacionales y climáticas uniformes. Sin embargo, se necesitan estudios adicionales en otras temporadas (i.e., evento El Niño Oscilación del Sur) que permitan tener una mejor aproximación en estas comunidades costeras.

Dentro del ensamble de tenebriónidos epigeos destacó Gyriosomus granulipennis como especie endémica de ECCh y PICh en la isla Choros, siendo una especie encontrada en baja frecuencia. Al respecto, Fattorini (2006) considera la importancia de la evaluación de los niveles de endemismo en sistemas insulares, al ser elementos con alta prioridad para la conservación (Pizarro-Araya et al., 2012). De esta manera la restringida distribución de ciertas especies dentro de los ecosistemas insulares puede ser considerada un indicador de rareza, criterio básico para identificar especies con necesidad de conservación.

\section{Agradecimientos}

Agradecemos a CONAF Región de Coquimbo (Paula Martínez y Pablo Arróspide) la ayuda en los permisos y facilidades para trabajar en la Reserva Nacional Pingüino de Humboldt (Proyectos N. ${ }^{\circ}$ 006/2014 y N. ${ }^{\circ}$ 028/2015). El presente estudio fue financiado por los proyectos FPA 04-015-2006, DIULS PR15121/VACDDI001 de la Universidad de La Serena, La Serena, Chile (JPA) y PIP 112-201101-00987 Consejo Nacional de Investigaciones Científicas y Técnicas (CONICET, Argentina) (GEF). Fermín M. Alfaro agradece a la beca CONICYT-PCHA/Magíster Nacional/2013-22130123.

\section{Referencias}

Aguirre, L. (1967). Geología de las islas Choros, Damas y de Punta Choros. Provincia de Coquimbo. Revista Minerales (Chile), 22, 73-83.

Alfaro, F. M., Pizarro-Araya, J. y Flores, G. E. (2009). Epigean tenebrionids (Coleoptera: Tenebrionidae) from the Choros Archipelago (Coquimbo Region, Chile). Entomological News, 120, 125-130.

Arancio, G. y Jara, P. (2007). Flora de la Reserva Nacional Pingüino de Humboldt. La Serena: Ediciones Universidad de La Serena.

Armesto, J. J., Vidiella, P. E. y Gutiérrez, J. R. (1993). Plant communities of the fog-free coastal desert of Chile: plant strategies in a fluctuating environment. Revista Chilena de Historia Natural, 66, 271-282.

Benítez, H. A., Pizarro-Araya, J., Bravi, R., Sanzana, M. J. y Alfaro, F. M. (2014). Morphological variation on isolated populations of Praocis (Praocis) spinolai. Journal of Insect Science, 14, 1-12.

Castro, C. y Brignardello, L. (2005). Geomorfología aplicada a la ordenación territorial de litorales arenosos: orientaciones para la protección, usos y aprovechamientos sustentables del sector de Los Choros, Comuna de La Higuera, IV Región. Revista de Geografía Norte Grande, 33, 33-58.

Cavieres, L. A., Arroyo, M. T. K., Posadas, P., Marticorena, C., Matthei, O., Rodríguez, R., et al. (2002). Identification of priority areas for conservation in an arid zone: application of parsimony analysis of endemicity in the vascular flora of the Antofagasta region, northern Chile. Biodiversity and Conservation, 11, 1303-1311.

Cavieres, L. A., Mihoc, M., Marticorena, A., Marticorena, C., Matthei, O. y Squeo, F. A. (2001). Determinación de áreas prioritarias de conservación: análisis de parsimonia de endemismos (PAE) en la flora de la IV Región de Coquimbo. En F. A. Squeo, G. Arancio, y J. R. Gutiérrez (Eds.), Libro rojo de la flora nativa y de los sitios prioritarios para su conservación: región de Coquimbo (pp. 159-170). La Serena: Ediciones Universidad de La Serena.

Cepeda-Pizarro, J., Pizarro-Araya, J. y Vásquez, H. (2005a). Composición y abundancia de artrópodos epigeos del Parque Nacional Llanos de Challe: impactos del ENOS de 1997 y efectos del hábitat pedológico. Revista Chilena de Historia Natural, 78, 635-650.

Cepeda-Pizarro, J., Pizarro-Araya, J. y Vásquez, H. (2005b). Variación en la abundancia de Arthropoda en un transecto latitudinal del desierto costero transicional de Chile, con énfasis en los tenebriónidos epigeos. Revista Chilena de Historia Natural, 78, 651-663.

Clarke, A. E. (1993). Vertical disintegration and spatial aspects of production subcontracting. Abstracts of the Association of American Geographers 1993 Annual Meeting, Atlanta, USA.

Cloudsley-Thompson, J. (2001). Thermal and water relations of desert beetles. Naturwissenschaften, 88, 447-460.

Cortés-Contreras, M., Pizarro-Araya, J., Alfaro, F. M. y Cepeda-Pizarro, J. (2013). Coleópteros epígeos (Insecta: Coleoptera) asociados a ecotopos dunarios de la cordillera de la costa de Chile. Idesia, 31, 95-101.

De Los Santos, A., de Nicolás, J. P. y Ferrer, F. (2002). Habitat selection and assemblage structure of darkling beetles (Col. Tenebrionidae) along environmental gradients on the Island of Tenerife (Canary Islands). Journal of Arid Environments, 52, 63-85.

De Los Santos, A., Gómez-González, L. A., Alonso, C., Arbelo, C. D. y de Nicolás, J. P. (2000). Adaptive trends of darkling beetles (Col. Tenebrionidae) on environmental gradients on the island of Tenerife (Canary Islands). Journal of Arid Environments, 45, 85-98.

Di Castri, F. y Hajek, E. R. (1976). Bioclimatología de Chile. Santiago: Editorial Universidad Católica de Chile.

Duncan, F. D., Dickman, C. R. y Christopher, R. (2009). Respiratory strategies of tenebrionid beetles in arid Australia: Does physiology beget nocturnality? Physiological Entomology, 34, 52-60.

Elgueta, M. (2013). Geosphaeropterus, nuevo género de Tropiphorini (Coleoptera: Curculionidae) de Chile, con descripción de tres nuevas especies. Boletín del Museo Nacional de Historia Natural, Chile, 62, 203-217.

Faith, D. P., Minchin, P. R. y Belbin, L. (1987). Compositional dissimilarity as a robust measure of ecological distance. Vegetatio, 69, 57-68.

Fattorini, S. (2002). Biogeography of the tenebrionid beetles (Coleoptera, Tenebrionidae) on the Aegean Islands (Greece). Journal of Biogeography, 29, 49-67. 
Fattorini, S. (2006). Detecting biodiversity hotspots by species-area relationships: a case study of Mediterranean beetles. Conservation Biology, 4, 1169-1180

Fattorini, S. (2009). Darkling beetle communities in two geologically contrasting biotopes: testing biodiversity patterns by microsite comparisons. Biological Journal of the Linnean Society, 98, 787-793.

Field, J. G., Clarke, K. y Warwick, R. (1982). A practical strategy for analyzing multispecies distributions. Marine Ecology Progress Series, 8, 37-52.

Flores, G. E. y Pizarro-Araya, J. (2012). Systematic revision of the South American genus Praocis Eschscholtz, 1829 (Coleoptera: Tenebrionidae). Part 1: Introduction and subgenus Praocis s. str. Zootaxa, 3336, 1-35.

Flores, G. E. y Pizarro-Araya, J. (2014). Towards a revision of the South American genus Praocis Eschscholtz (Coleoptera: Tenebrionidae), with estimation of the diversity of each subgenus. ZooKeys, 415, 53-80.

Gajardo, R. (1993). La vegetación natural de Chile. Santiago, Chile: Editorial Universitaria.

Gaston, K. J. (2000). Global patterns in biodiversity. Nature, 405, 220-227.

Gotelli, N. J. y Colwell, R. K. (2010). Estimating species richness. En A. E. Magurran y B. J. McGill (Eds.), Biological diversity: frontiers in measurement and assessment (pp. 39-54). Oxford: Oxford University Press.

Grismado, C. J. y Pizarro-Araya, J. (2016). The spider genus Cyrioctea Simon on Isla Chañaral (Pingüino de Humboldt National Reserve, Atacama, Chile): Description of a new species, and description of the male of Cyrioctea cruz Platnick (Araneae, Zodariidae). Zootaxa, 107, 267-276.

Hammer, O., Harper, D. y Ryan, P. (2001). PAST: Paleontological statistics software for education and data analysis. Paleontología Electrónica, 4, 1-9.

Hausdorf, B. y Henning, C. (2005). The influence of recent geography, palaeogeography and climate on the composition of the fauna of the central Aegean Islands. Biological Journal of the Linnean Society, 84, 785-795.

Jerez, V. (2000). Diversidad y patrones de distribución geográfica de insectos coleópteros en ecosistemas desérticos de la región de Antofagasta, Chile. Revista Chilena de Historia Natural, 73, 79-92.

Johnson, R. y Wichern, D. (1992). Applied multivariate statistical analysis. New York: Prentice Hall.

Kruskal, J. B. (1964). Multidimensional scaling by optimizing goodness of fit to a nonmetric hypothesis. Psychometrika, 29, 1-27.

Matthews, E. G., Lawrence, J. F., Bouchard, P., Steiner, W. E. y Ślipiński, S. A. (2010). Tenebrionidae Latreille, 1802. En R. A. B. Leschen, R. G. Beutel, y J. F. Lawrence (Eds.), Handbook of zoology. Coleoptera, Beetles. Vol. 2. Morphology and systematics (Elateroidea, Bostrichiformia partim) (pp. 574-659). Berlín y Nueva York: De Gruyter.

Ministerio de Medio Ambiente. (2011). Clasificación de especies del 7o proceso. Recuperado el 23 de septiembre, 2016 de: http://www mma.gob.cl/clasificacionespecies/fichas7proceso/fichas_pac/Gyriosomus_ granulipennis_P07.pdf.

Morrone, J. J. (2015). Biogeographical regionalisation of the Andean region. Zootaxa, 3936, 207-236.
Novoa, R. y Villaseca, S. (1989). Mapa agroclimático de Chile. Santiago: Instituto de Investigaciones Agropecuarias.

Pan, C. C., Zhao, H. L., Feng, Q., Liu, J. L., Liu, L. D., Cai, Y. J., et al. (2015). Temporal variations of ground-dwelling arthropods in relation to grassland salinization. European Journal of Soil Biology, 68, 25-32.

Parmenter, R. R. y MacMahon, J. A. (1984). Factors influencing the distribution and abundance of ground dwelling beetles (Coleoptera) in a shrub-steppe ecosystem: the role of shrub architecture. Pedobiologia, 26, 21-34.

Parmenter, R. R., Parmenter, Ch. A. y Cheney, C. D. (1989). Factors influencing partitioning in arid-land darkling beetles (Tenebrionidae): temperature and winter conservation. Journal of Arid Environments, 17, 57-67.

Pizarro-Araya, J. (2010). Hábitos alimenticios del género Gyriosomus GuérinMéneville, 1834 (Coleoptera: Tenebrionidae): ¿Qué comen las vaquitas del desierto costero? Idesia, 28, 115-119.

Pizarro-Araya, J., Agusto, P., López-Cortés, F., Ojanguren-Affilastro, A. A., Briones, R. y Cepeda-Pizarro, J. (2014). Diversidad y composición estacional de la escorpiofauna (Arachnida: Scorpiones) del archipiélago Los Choros (Región de Coquimbo, Chile). Gayana, 78, 46-56.

Pizarro-Araya, J., Alfaro, F. M., Cortés-Contreras, M., Rivera, C., VargasTalciani, P. y Ojanguren-Affilastro, A. A. (2014). Epigean insects of Chañaral Island (Pingüino de Humboldt National Reserve, Atacama, Chile). Journal of the Entomological Research Society, 16, 39-50.

Pizarro-Araya, J., Cepeda-Pizarro, J. y Flores, G. E. (2008). Diversidad taxonómica de los artrópodos epígeos de la región de Atacama (Chile): estado del conocimiento. En F. A. Squeo, G. Arancio, y J. R. Gutiérrez (Eds.), Libro Rojo de la flora nativa y de los sitios prioritarios para su conservación: región de Atacama (pp. 257-274). La Serena: Ediciones Universidad de La Serena.

Pizarro-Araya, J. y Flores, G. E. (2004). Two new species of Gyriosomus Guérin-Méneville from Chilean coastal desert (Coleoptera: Tenebrionidae: Nycteliini). Journal of the New York Entomological Society, 112, 121-126.

Pizarro-Araya, J., Vergara, O. E. y Flores, G. E. (2012). Gyriosomus granulipennis (Coleoptera: Tenebrionidae) un caso extremo a conservar. Revista Chilena de Historia Natural, 85, 345-349.

SAG (Servicio Agrícola y Ganadero). (2015). La ley de caza y su reglamento. Santiago: División de Protección de los Recursos Naturales Renovables, SAG.

Shannon, C. E. y Weaver, W. (1949). The mathematical theory of communication. Urbana, IL: University of Illinois.

Valdivia, D. E., Pizarro-Araya, J., Briones, R., Ojanguren-Affilastro, A. A. y Cepeda-Pizarro, J. (2011). Taxonomical diversity and abundance of solpugids (Arachnida: Solifugae) in coastal ecotopes of north-central Chile. Revista Mexicana de Biodiversidad, 82, 1234-1242.

Vidal, M. A., Pizarro-Araya, J., Jerez, V. y Ortiz, J. C. (2011). Daily activity and thermoregulation in predatore-prey interaction during the Flowering Desert in Chile. Journal of Arid Environments, 75, 802-808. 